\title{
A Global Bifurcation Theorem for Convex-Valued Differential Inclusions
}

\author{
S. Domachowski and J. Gulgowski
}

Abstract. In this paper we prove a global bifurcation theorem for convex-valued completely continuous maps. Basing on this theorem we prove an existence theorem for convex-valued differential inclusions with Sturm-Liouville boundary conditions

$$
\left.\begin{array}{rl}
u^{\prime \prime}(t) & \in \varphi\left(t, u(t), u^{\prime}(t)\right) \text { for a.e. } t \in(a, b) \\
l(u) & =0
\end{array}\right\} .
$$

The assumptions refer to the appropriate asymptotic behaviour of $\varphi(t, x, y)$ for $|x|+$ $|y|$ close to 0 and to $+\infty$, and they are independent from the well known Bernsteintype conditions. In the last section we give a set of examples of $\varphi$ satisfying the assumptions of the given theorem but not satisfying the Bernstein conditions.

Keywords: Differential inclusions, Sturm-Liouville boundary conditions, global bifurcation

MSC 2000: Primary 47H04, secondary 34A60, 34B24

\section{Global bifurcation theorem}

Let $E$ be a real Banach space, $A \subset \mathbb{R}$ an open interval and $c f(E)$ the family of all non-empty, closed, bounded and convex subsets of $E$. We call a map $F: A \times E \rightarrow c f(E)$ completely continuous if $F$ is upper semicontinuous and, for any bounded set $B \subset A \times E$, the set $F(B) \subset E$ is relatively compact.

Let $F: A \times E \rightarrow c f(E)$ be a completely continuous map such that $0 \in F(\lambda, 0)$ for $\lambda \in A$ and let $f: A \times E \rightarrow c f(E)$ be given by

$$
f(\lambda, x)=x-F(\lambda, x)
$$

We call $\left(\mu_{0}, 0\right) \in A \times E$ a bifurcation point of the map $f$ if for all open subsets $U \subset A \times E$ with $\left(\mu_{0}, 0\right) \in U$ there exists a point $(\lambda, x) \in U$ such that $x \neq 0$ and $0 \in f(\lambda, x)$. Let us denote the set of all bifurcation points of $f$ by $\mathcal{B}_{f}$.

Both authors: University of Gdańsk, Institute of Mathematics, Wita Stwosza 57, PL-80-952 Gdańsk, Poland; mdom@math.univ.gda.pl

ISSN 0232-2064 / \$2.50 C Heldermann Verlag Berlin 
Let $\mathcal{R}_{f} \subset A \times E$ be the closure (in $A \times E$ ) of the set of non-trivial solutions of the inclusion $0 \in f(\lambda, x)$, i.e.

$$
\mathcal{R}_{f}=\overline{\{(\lambda, x) \in A \times E: x \neq 0 \text { and } 0 \in f(\lambda, x)\}} .
$$

Let us observe that, for each $(\lambda, x) \in \mathcal{R}_{f}, 0 \in f(\lambda, x)$.

Let $U \subset E$ be a bounded open subset and let the map $g: \bar{U} \rightarrow c f(E)$ be given by $g(x)=x-G(x)$, where $G: \bar{U} \rightarrow c f(E)$ is a completely continuous map such that, for $x \in \partial U$, the relation $x \notin G(x)$ holds. It is well known that in such situation we may define the Leray-Schauder $\operatorname{degree} \operatorname{deg}(g, U, 0)$ (cf. [2, $3,8,17,19])$.

For each $\lambda$ satisfying $(\lambda, 0) \notin \mathcal{B}_{f}$ there exists an $r_{0}>0$ such that, for $\|x\|=$ $r \in\left(0, r_{0}\right]$, the relation $x \notin F(\lambda, x)$ holds. So the value $\operatorname{deg}(f(\lambda, \cdot), B(0, r), 0)$ is defined. Assume that for an interval $[a, b] \subset A$ there exists a $\delta>0$ such that

$$
(([a-\delta, a) \cup(b, b+\delta]) \times\{0\}) \cap \mathcal{B}_{f}=\emptyset .
$$

Then we may define the bifurcation index $s[f, a, b]$ of the map $f$ with respect to the interval $[a, b]$ as

$$
s[f, a, b]=\lim _{\lambda \rightarrow b^{+}} \operatorname{deg}(f(\lambda, \cdot), B(0, r), 0)-\lim _{\lambda \rightarrow a^{-}} \operatorname{deg}(f(\lambda, \cdot), B(0, r), 0)
$$

where $r=r(\lambda)>0$ is small enough.

Now we are going to give some auxiliary lemmas, which will be used in the proof of the global bifurcation theorem below. We are going to use a separation lemma for closed subsets of compact Hausdorff spaces given in [9] (see also [24: Section XI]).

Lemma 1. Assume that $X, Y$ are closed subsets of a compact Hausdorff space $K$ and that there does not exist a connected set $S \subset K$ such that $S \cap X \neq \emptyset$ as well as $S \cap Y \neq \emptyset$. Then there exists a separation $K=K_{x} \cup K_{y}$ with $K_{x} \cap K_{y}=\emptyset$ such that $X \subset K_{x}$ and $Y \subset K_{y}$ and both $K_{x}$ and $K_{y}$ are open and closed in $K$.

An immediate consequence of Lemma 1 is the following

Proposition 1. Let the map $f: A \times E \rightarrow c f(E)$ be given by (1.1) and let $[a, b] \subset A$ be an interval such that $([a, b] \times\{0\}) \cap \mathcal{B}_{f} \neq \emptyset$. Further, let $\mathcal{C}_{0}$ be $a$ compact component of the set $\mathcal{R}=\mathcal{R}_{f} \cup([a, b] \times\{0\})$ such that $[a, b] \times\{0\} \subset \mathcal{C}_{0}$. Then there exists an open and closed set $\mathcal{K}_{0} \subset \mathcal{R}$ such that

$$
\mathcal{C}_{0} \subset \mathcal{K}_{0} \subset(c, d) \times B(0, R) \subset[c, d] \times \overline{B(0, R)} \subset A \times E
$$


Now we are going to give a generalization of Ize's lemma (cf. [14] and [20: Lemma 3.4.2]) to convex-valued completely continuous vector fields. For this let the function $\rho(\cdot,[a, b]): \mathbb{R} \rightarrow[0,+\infty)$ be given by

$$
\rho(\lambda,[a, b])= \begin{cases}a-\lambda & \text { for } \lambda<a \\ 0 & \text { for } \lambda \in[a, b] \\ \lambda-b & \text { for } \lambda>b .\end{cases}
$$

Lemma 2. Let the map $f: A \times E \rightarrow c f(E)$ be given by (1.1) and let $[a, b] \subset A$ be an interval such that $\mathcal{B}_{f} \subset[a, b] \times\{0\}$. Then there exists an $\varepsilon_{0}>0$ such that for all $\varepsilon \in\left(0, \varepsilon_{0}\right]$ there is an $r_{0}>0$ so that the map

$$
\begin{aligned}
& f_{r, \varepsilon}: \overline{U_{r, \varepsilon}} \rightarrow c f(\mathbb{R} \times E) \\
& f_{r, \varepsilon}(\lambda, x)=\left\{\left(\|x\|^{2}-r^{2}, y\right): y \in f(\lambda, x)\right\}
\end{aligned}
$$

with

$$
U_{r, \varepsilon}=\left\{(\lambda, x) \in \mathbb{R} \times E:\|x\|^{2}+\rho^{2}(\lambda,[a, b])<r^{2}+\varepsilon^{2}\right\}
$$

is a completely continuous vector field and

$$
\operatorname{deg}\left(f_{r, \varepsilon}, U_{r, \varepsilon}, 0\right)=-s[f, a, b] \quad\left(r \in\left(0, r_{0}\right]\right) .
$$

The proof of the lemma is a modification of that given in [12] for the single-valued case and $[a, b]=\left\{\lambda_{0}\right\}$. It is enough to replace the function $d(\lambda)=\left|\lambda-\lambda_{0}\right|$ by $d(\lambda)=\rho(\lambda,[a, b])$. For an overview of this technique see also [15: Remark 1.5].

Theorem 1. Let the map $f: A \times E \rightarrow c f(E)$ be given by (1.1) and assume that there exists an interval $[a, b] \subset A$ such that $\mathcal{B}_{f} \subset[a, b] \times\{0\}$ and $s[f, a, b] \neq$ 0 . Then there exists a non-compact component $\mathcal{C} \subset \mathcal{R}_{f}$ satisfying $\mathcal{C} \cap \mathcal{B}_{f} \neq \emptyset$.

Proof. As consequence of the homotopy property of the topological degree and $s[f, a, b] \neq 0$ we have $([a, b] \times\{0\}) \cap \mathcal{B}_{f} \neq \emptyset$. Let $\mathcal{C}_{0}$ be a component of the set $\mathcal{R}=\mathcal{R}_{f} \cup([a, b] \times\{0\})$ such that $[a, b] \times\{0\} \subset \mathcal{C}_{0}$. Assume further that $\mathcal{C}_{0}$ is compact. By Proposition 1 there exists a bounded open and closed set $\mathcal{K} \subset \mathcal{R}$ such that $\mathcal{C}_{0} \subset \mathcal{K}$. So there exists a bounded and open set $U \subset A \times E$ satisfying $\mathcal{K} \subset U$ and $(\mathcal{R} \backslash \mathcal{K}) \cap \bar{U}=\emptyset$. Hence for $(\lambda, x) \in \partial U$ and $r>0$ we have $0 \notin f_{r}(\lambda, x)$. Moreover, for any $r_{1}, r_{2}>0$ the maps $f_{r_{1}}$ and $f_{r_{2}}$ may be joined by homotopy. We can see as well that for large $R>0$ the map $f_{R}$ has no zeroes in $\bar{U}$ so that $\operatorname{deg}\left(f_{r}, U, 0\right)=0$ for $r>0$. There exist $\varepsilon>0$ and $r_{1}>0$ such that $\overline{U_{r_{1}, \varepsilon}} \subset U$. Further, by Lemma 2 there exists $r^{\prime} \in\left(0, r_{1}\right]$ such that $\operatorname{deg}\left(f_{r^{\prime}}, U_{r^{\prime}, \varepsilon}, 0\right)=-s[f, a, b]$. Of course, $\overline{U_{r^{\prime}, \varepsilon}} \subset U$.

Because $\mathcal{B}_{f} \subset[a, b] \times\{0\}$ and $U$ is bounded, there exists a number $r_{2}>0$ such that $0 \notin f(\lambda, x)$ for $(\lambda, x) \in U$ with $0<\|x\| \leq r_{2}$ and $\rho(\lambda,[a, b]) \geq \varepsilon$. 
Let $r \in\left(0, \min \left\{r^{\prime}, r_{2}\right\}\right)$. Then $\overline{U_{r, \varepsilon}} \subset U$. Hence, if $0 \in f_{r}(\lambda, x)$ then $\|x\|=$ $r<r_{2}$ and $\rho(\lambda,[a, b])<\varepsilon$. Then we have $\|x\|^{2}+\rho^{2}(\lambda,[a, b])<r^{2}+\varepsilon^{2}$ and $(\lambda, x) \in U_{r, \varepsilon}$. Consequently, we have the implication

$$
(\lambda, x) \in \bar{U} \backslash U_{r, \varepsilon} \quad \Longrightarrow \quad 0 \notin f_{r}(\lambda, x)
$$

That is why we have $\operatorname{deg}\left(f_{r}, U_{r, \varepsilon}, 0\right)=\operatorname{deg}\left(f_{r}, U, 0\right)$ and the contradiction

$$
0=\operatorname{deg}\left(f_{r}, U, 0\right)=\operatorname{deg}\left(f_{r}, U_{r, \varepsilon}, 0\right)=-s[f, a, b] \neq 0 .
$$

Because of this contradiction there exists a non-compact component $\mathcal{C}_{0} \subset$ $\mathcal{R}_{f} \cup([a, b] \times\{0\})$. What we are going to prove now is that there exists a noncompact component $\mathcal{C}$ of $\mathcal{R}_{f}$ such that $\mathcal{C} \cap \mathcal{B}_{f} \neq \emptyset$. Of course, such component has to satisfy $\mathcal{C} \subset \mathcal{C}_{0}$.

At the beginning let us denote by $\Gamma$ the family of all components $\gamma$ of $\mathcal{R}_{f}$ such that $\gamma \cap \mathcal{B}_{f} \neq \emptyset$. Further, let $G=\bigcup_{\gamma \in \Gamma} \gamma$. We can observe that $G \subset \mathcal{C}_{0}$. We are going to show that there exists a $\gamma \in \Gamma$ such that $\gamma$ is not compact. But first assume, contrary to our claim, that each $\gamma \in \Gamma$ is compact.

Let us now take $B=(c, d) \times B(0, R)$ such that

$$
[a, b] \times\{0\} \subset B \subset \bar{B} \subset A \times E,
$$

let us denote by $\Gamma_{B}$ the family of all that components $\gamma$ of $\mathcal{R}_{f} \cap \bar{B}$ for which $\gamma \cap \mathcal{B}_{f} \neq \emptyset$ and let us also denote $G_{B}=\bigcup_{\gamma \in \Gamma_{B}} \gamma$. We can see that $\mathcal{B}_{f} \subset G_{B}$. We are going to show that $G_{B}$ is a closed subset of $\mathcal{R}_{f} \cap \bar{B}$. For this let $\left\{\left(\lambda_{n}, x_{n}\right)\right\} \subset G_{B}$ be a sequence such that $\left(\lambda_{n}, x_{n}\right) \rightarrow\left(\lambda_{0}, x_{0}\right) \in \mathcal{R}_{f} \cap \bar{B}$ and let $\gamma_{n} \in \Gamma_{B}$ be such that $\left(\lambda_{n}, x_{n}\right) \in \gamma_{n}$. Assume, contrary to our claim, that $\left(\lambda_{0}, x_{0}\right) \notin G_{B}$. Then $x_{0} \neq 0$ and the component $\gamma_{0}$ of $\mathcal{R}_{f} \cap \bar{B}$ containing $\left(\lambda_{0}, x_{0}\right)$ is such that $\gamma_{0} \cap \mathcal{B}_{f}=\emptyset$. In this case we may apply Lemma 1 to the case of $K=\mathcal{R}_{f} \cap \bar{B}, X=\left\{\left(\lambda_{0}, x_{0}\right)\right\}$ and $Y=\mathcal{B}_{f}$. Then there exist sets $K_{x}, K_{y} \subset K$ open and closed in $K$ such that

$$
\left(\lambda_{0}, x_{0}\right) \in K_{x}, \quad \mathcal{B}_{f} \subset K_{y}, \quad K_{x} \cap K_{y}=\emptyset, \quad K=K_{x} \cup K_{y} .
$$

Because for large $n \in \mathbb{N}$ the relation $\gamma_{n} \cap K_{x} \neq \emptyset$ holds and $\gamma_{n} \cap K_{y} \neq \emptyset$, this contradicts the connectedness of $\gamma_{n}$.

Now we are going to consider the following two situations:

(i) There exists $B_{0}=(c, d) \times B(0, R)$ such that $[a, b] \times\{0\} \subset B_{0} \subset \overline{B_{0}} \subset$ $A \times E$ and $G \subset B_{0}$.

(ii) There exists a sequence $\left\{\gamma_{n}\right\} \subset \Gamma$ such that, for each $B=(c, d) \times$ $B(0, R)$ satisfying $[a, b] \times\{0\} \subset B \subset \bar{B} \subset A \times E$, the relation $\gamma_{n} \not \subset \bar{B}$ holds for $n \in \mathbb{N}$ large enough. 
Let us first assume that (i) holds and let $C_{0}^{B_{0}}$ be a component of $\mathcal{C}_{0} \cap \overline{B_{0}}$ such that $[a, b] \times\{0\} \subset C_{0}^{B_{0}}$. Of course, we have $G_{B_{0}} \subset C_{0}^{B_{0}}$. By Lemma 1 , in this case $C_{0}^{B_{0}} \subset B_{0}$ and there must be also $\mathcal{C}_{0} \subset B_{0}$, what contradicts that $\mathcal{C}_{0}$ is not compact. So we can assume that there exists $\left(\lambda_{0}, x_{0}\right) \in \partial B_{0} \cap C_{0}^{B_{0}}$. We can apply Lemma 1 for $K=\mathcal{R}_{f} \cap \overline{B_{0}}, X=\left\{\left(\lambda_{0}, x_{0}\right)\right\}$ and $Y=\mathcal{B}_{f}$. Because $\left(\lambda_{0}, x_{0}\right) \notin G_{B_{0}}$, there does not exist a component $\gamma$ of $K$ such that $\left(\lambda_{0}, x_{0}\right) \in \gamma$ and $\gamma \cap \mathcal{B}_{f} \neq \emptyset$. Then by Lemma 1 , there exist open and closed sets $K_{x}, K_{y} \subset K$ such that

$$
\left(\lambda_{0}, x_{0}\right) \in K_{x}, \quad \mathcal{B}_{f} \subset K_{y}, \quad K_{x} \cap K_{y}=\emptyset, \quad K_{x} \cup K_{y}=K .
$$

This implies that there exist an $r>0$ such that $K_{x} \cap([a, b] \times \overline{B(0, r)})=\emptyset$. Hence

$$
\begin{aligned}
& K_{x} \cap\left(K_{y} \cup([a, b] \times\{0\})\right)=\emptyset \\
& K_{x} \cup\left(K_{y} \cup([a, b] \times\{0\})\right)=K \cup([a, b] \times\{0\})
\end{aligned}
$$

and both $K_{x}$ and $K_{y} \cup([a, b] \times\{0\})$ are open and closed in $K \cup([a, b] \times\{0\})$. But the set $C_{0}^{B_{0}} \subset K \cup([a, b] \times\{0\})$ is connected and

$$
\begin{array}{r}
C_{0}^{B_{0}} \cap K_{x} \neq \emptyset \\
C_{0}^{B_{0}} \cap\left(K_{y} \cup([a, b] \times\{0\}) \neq \emptyset\right.
\end{array}
$$

what gives the contradiction.

In this case the situation (ii) holds true. Let us fix any $B$ as given in (ii) and let $\tilde{\gamma}_{n} \in \Gamma_{B}$ be such that $\tilde{\gamma}_{n} \subset \gamma_{n}$ and $\left(\lambda_{n}, x_{n}\right) \in \tilde{\gamma}_{n} \cap \partial B$. Because $x_{n} \in F\left(\lambda_{n}, x_{n}\right)$, we may assume that there exists a subsequence of $\left(\lambda_{n}, x_{n}\right)$ converging to $\left(\lambda_{0}, x_{0}\right)$. As we observed before, $\left(\lambda_{0}, x_{0}\right) \in G_{B}$. So there exists a component $\tilde{\gamma}_{0} \in \Gamma_{B}$ such that $\left(\lambda_{0}, x_{0}\right) \in \tilde{\gamma}_{0}$. Let $\gamma_{0} \in \Gamma$ be such that $\tilde{\gamma}_{0} \subset \gamma_{0}$. From our general assumption $\gamma_{0}$ is compact. By Proposition 1 there exists an open and closed set $K \subset \mathcal{R}_{f}$ such that $\gamma_{0} \subset K \subset B_{0}$ for some $B_{0}=(c, d) \times B\left(0, R_{0}\right)$ so that $B_{0} \subset \overline{B_{0}} \subset A \times E$. But for $n \in \mathbb{N}$ large enough the relations $K \cap \gamma_{n} \neq \emptyset$ and $\gamma_{n} \not \subset B_{0}$ hold. This gives $\gamma_{n} \cap K \neq \emptyset$ and $\gamma_{n} \cap\left(\mathcal{R}_{f} \backslash K\right) \neq \emptyset$, what contradicts the connectedness of $\gamma_{n}$.

So both (i) and (ii) cannot hold what implies that there exists $\gamma \in \Gamma$ which is not compact.

The existence of components (in the single-valued case) emanating from bifurcation points was studied by Krasnoselskii (see [16]). The global bifurcation theorem for the single-valued case was proved by Rabinowitz in [23] (see also [9]) in the following version:

Theorem A. Let $L: E \rightarrow E$ be a compact linear map, let $H: \mathbb{R} \times E \rightarrow E$ be a compact and continuous map such that $H(\lambda, u)=o(\|u\|)$ for $u$ near 
0 uniformly on bounded $\lambda$ intervals, and let the map $f: \mathbb{R} \times E \rightarrow E$ be given by $f(\lambda, u)=u-\lambda L(u)-H(\lambda, u)$. Then, if $\mu$ is an eigenvalue of $L$ of odd multiplicity, then $\mathcal{R}_{f}$ posseses a maximal subcontinuum $\mathcal{C}_{\mu}$ such that $(\mu, 0) \in \mathcal{C}_{\mu}$ and $\mathcal{C}_{\mu}$ either

(i) meets infinity in $\mathbb{R} \times E$

or

(ii) meets $(\hat{\mu}, 0)$, where $\mu \neq \hat{\mu}$ and $\hat{\mu}$ is an eigenvalue of $L$.

The proof of Theorem 1 follows the ideas of complementing the map introduced by Ize (see [14], but also [20: Section 3.4]). The original version of the Rabinowitz theorem found numerous generalizations and modifications (for an overview see $[4,15]$ ). The single-valued version of the global bifurcation theorem is probably most similar to what is proved in [18: Theorem 2.5]. Theorem 1 is not only a generalization of [18: Theorem 2.5] to convex-valued maps, but also gives stronger results (it gives the existence of the component of $\mathcal{R}_{f}$ instead of the component of $\left.\mathcal{R}_{f} \cup([a, b] \times\{0\})\right)$.

The convex-valued case was already considered by the authors in [1] for a much more general situation of parameter space of dimension greater than 1. The authors gave there sufficient conditions for the existence of a global bifurcation branch emanating from $(0,0)$. In Theorem 1 we focus on the case of scalar parameters but, on the other hand, we do not assume that the bifurcation points are isolated in the set of all bifurcation points.

\section{Existence theorem for convex-valued differential inclusion}

In this section we need the following notations. For $x=\left(x_{1}, \ldots, x_{k}\right) \in \mathbb{R}^{k}$ we write $|x|=\sum_{i=1}^{k}\left|x_{i}\right|$ and call $x$ non-negative (and write $x \geq 0$ ) when $x_{1}, \ldots, x_{k} \geq 0$. Let the map $p: \mathbb{R}^{k} \rightarrow \mathbb{R}^{k}$ be given by

$$
p\left(x_{1}, \ldots, x_{k}\right)=\left(\eta_{1}\left|x_{1}\right|, \ldots, \eta_{k}\left|x_{k}\right|\right)
$$

where $\eta_{1}, \ldots, \eta_{k} \geq 0$ and $\eta_{1}^{2}+\ldots+\eta_{k}^{2}>0$, let $\|\cdot\|_{0}$ be the supremum norm in $C[a, b]$ and let $\|\cdot\|_{k}$ be the norm in $C^{1}\left([a, b], \mathbb{R}^{k}\right)$ given by $\|u\|_{k}=\sum_{i=1}^{k}\left(\left\|u_{i}\right\|_{0}+\right.$ $\left.\left\|u_{i}^{\prime}\right\|_{0}\right)$ for $u=\left(u_{1}, \ldots, u_{k}\right) \in C^{1}\left([a, b], \mathbb{R}^{k}\right)$.

Let us recall that a multi-valued map $\varphi:[a, b] \times \mathbb{R}^{k} \times \mathbb{R}^{k} \rightarrow c f\left(\mathbb{R}^{k}\right)$ is a Carathéodory map if the map $\varphi(\cdot, x, y):[a, b] \rightarrow c f\left(\mathbb{R}^{k}\right)$ is measurable for all $(x, y) \in \mathbb{R}^{2 k}$, the map $\varphi(t, \cdot, \cdot): \mathbb{R}^{2 k} \rightarrow \operatorname{cf}\left(\mathbb{R}^{k}\right)$ is upper semicontinuous for all $t \in[a, b]$, and for each $R>0$ there exists an integrable function $m_{R} \in L^{1}(a, b)$ such that

$$
\left\{\begin{array}{l}
\forall w \in L^{1}\left((a, b), \mathbb{R}^{k}\right) \\
\forall(x, y) \in \mathbb{R}^{2 k} \\
\forall t \in[a, b]
\end{array}\right\}: \quad\left\{\begin{array}{l}
|x|+|y| \leq R \\
w(t) \in \varphi(t, x, y)
\end{array}\right\} \Rightarrow|w(t)| \leq m_{R}(t) .
$$


In this section we will give sufficient conditions for the existence of the solution of the boundary value problem

$$
\left.\begin{array}{rl}
u^{\prime \prime}(t) & \in \varphi\left(t, u(t), u^{\prime}(t)\right) \text { for a.e. } t \in(a, b) \\
l(u) & =0
\end{array}\right\}
$$

where $\varphi:[a, b] \times \mathbb{R}^{k} \times \mathbb{R}^{k} \rightarrow c f\left(\mathbb{R}^{k}\right)$ is a Carathéodory map and the map $l: C^{1}\left([a, b], \mathbb{R}^{k}\right) \rightarrow \mathbb{R}^{k} \times \mathbb{R}^{k}$ is given by

$$
l\left(u_{1}, \ldots, u_{k}\right)=\left(l_{1}\left(u_{1}\right), \ldots, l_{k}\left(u_{k}\right)\right)
$$

where $l_{j}\left(u_{j}\right)=\left(u_{j}(a) \sin \alpha_{j}-u_{j}^{\prime}(a) \cos \alpha_{j}, u_{j}(b) \sin \beta_{j}+u_{j}^{\prime}(b) \cos \beta_{j}\right)$ with $\alpha_{j}, \beta_{j} \in\left[0, \frac{\pi}{2}\right]$ and $\alpha_{j}^{2}+\beta_{j}^{2}>0 \quad(j=1, \ldots, k)$. It is well known (cf. [13: Theorem XI.4.1]) that with the boundary value problem

$$
\left.\begin{array}{l}
u_{i}^{\prime \prime}(t)=h_{i}(t) \text { for a.e. } t \in(a, b) \\
l_{i}\left(u_{i}\right)=0
\end{array}\right\}
$$

we may associate a continuous map $T_{i}: L^{1}(a, b) \rightarrow C^{1}[a, b]$ such that $T_{i}\left(h_{i}\right)=$ $u_{i}$ if and only if $u_{i} \in C^{1}[a, b], u_{i}^{\prime}:[a, b] \rightarrow \mathbb{R}^{1}$ is absolutely continuous and $u_{i}$ is a solution of problem (2.3).

Consider the map

$$
\begin{aligned}
& T: L^{1}\left((a, b), \mathbb{R}^{k}\right) \rightarrow C^{1}\left([a, b], \mathbb{R}^{k}\right) \\
& T\left(u_{1}, \ldots, u_{k}\right)=\left(T_{1} u_{1}, \ldots, T_{k} u_{k}\right) .
\end{aligned}
$$

We can see that

$$
u=T h \Longleftrightarrow\left\{\begin{aligned}
u^{\prime \prime}(t) & =h(t) \text { for a.e. } t \in(a, b) \\
l(u) & =0
\end{aligned}\right.
$$

for $h \in L^{1}\left((a, b), \mathbb{R}^{k}\right)$. The map $T$ has the following properties:

- For the Niemytzki operator $\Phi: C^{1}\left([a, b], \mathbb{R}^{k}\right) \rightarrow c f\left(L^{1}\left((a, b), \mathbb{R}^{k}\right)\right)$ associated with $\varphi$ and given by

$$
\Phi(u)=\left\{w \in L^{1}\left((a, b), \mathbb{R}^{k}\right): w(t) \in \varphi\left(t, u(t), u^{\prime}(t)\right)\right\}
$$

the superposition $T \circ \Phi: C^{1}\left([a, b], \mathbb{R}^{k}\right) \rightarrow c f\left(C^{1}\left([a, b], \mathbb{R}^{k}\right)\right)$ is completely continuous (cf. [22: Proposition 3.6]). 
- For $u, v \in C\left([a, b], \mathbb{R}^{k}\right)$ such that $l(u)=l(v)=0$ we have

$$
\langle T u, v\rangle=\langle u, T v\rangle
$$

where $\langle u, v\rangle=\int_{a}^{b}\left(\sum_{i=1}^{k} u_{i}(t) v_{i}(t)\right) d t$ (cf. [13: Theorem XI.4.1]).

- (Maximum principle, cf. [21: Chapter 1/Theorem 2]) If the functions $u \in C^{2}\left([a, b], \mathbb{R}^{k}\right)$ and $h \in C\left([a, b], \mathbb{R}^{k}\right)$ satisfy

$$
\left.\begin{array}{rl}
u^{\prime \prime}(t) & =h(t) \text { for a.e. } t \in(a, b) \\
l(u) & =0
\end{array}\right\}
$$

and $h \leq 0$, then $u \geq 0$.

Before state the existence theorem we must refer to some spectral properties of the linear single-valued problem

$$
\left.\begin{array}{rl}
u^{\prime \prime}(t)+\lambda u(t) & =0 \text { for } t \in(a, b) \\
l(u) & =0
\end{array}\right\} .
$$

It is obvious that $\mu \in \mathbb{R}$ is an eigenvalue of problem (2.7) if and only if there exists $j \in\{1, \ldots, k\}$ such that $\mu$ is an eigenvalue of the scalar problem

$$
\left.\begin{array}{rl}
u_{j}^{\prime \prime}(t)+\lambda u_{j}(t) & =0 \text { for } t \in(a, b) \\
l_{j}\left(u_{j}\right) & =0
\end{array}\right\} .
$$

It is well known (cf [13: Theorem XI.4.1]) that there exists exactly one eigenvalue $\mu_{j} \in \mathbb{R}$ of problem $(2.7)_{j}$, for which there exists an eigenvector $v_{\mu_{j}}$ such that $v_{\mu_{j}}(t)>0$ for $t \in(a, b)$, and then $\mu_{j}>0$. Let us observe that then $u_{\mu_{j}}=\left(0, \ldots, v_{\mu_{j}}, \ldots 0\right)$ is the eigenvector of problem (2.7) associated with the eigenvalue $\mu_{j}$.

Lemma 3. Assume that $(\lambda, u) \in(0,+\infty) \times C^{1}\left([a, b], \mathbb{R}^{k}\right)$ is a solution of the problem

$$
\left.\begin{array}{rl}
u^{\prime \prime}(t)+\lambda p(u(t)) & =0 \quad \text { for } t \in(a, b) \\
l(u) & =0
\end{array}\right\}
$$

and $u \neq 0$. Then $\lambda \in \Lambda=\left\{\frac{\mu_{i}}{\eta_{i}}: \eta_{i}>0\right\}$.

Proof. Let us first observe that $\Lambda \neq \emptyset$. By the maximum principle, for each $(\lambda, u) \in(0,+\infty) \times C^{1}\left([a, b], \mathbb{R}^{k}\right)$ being a solution of problem (2.8) we have $u \geq 0$. So, for $i=1, \ldots, k$,

$$
\left.\begin{array}{rl}
u_{i}^{\prime \prime}(t)+\lambda \eta_{i} u_{i}(t) & =0 \text { for } t \in(a, b) \\
l_{i}\left(u_{i}\right) & =0 \\
u_{i} & \geq 0
\end{array}\right\} .
$$

If $\eta_{i}=0$, then there must be $u_{i}=0$. On the other hand, for $\eta_{i}>0$ the only $\lambda>0$ for which $u \neq 0$ equals $\lambda=\frac{\mu_{i}}{\eta_{i}}$. 
Before we state the existence theorem let us assume that a Carathéodory $\operatorname{map} \varphi:[a, b] \times \mathbb{R}^{k} \times \mathbb{R}^{k} \rightarrow c f\left(\mathbb{R}^{k}\right)$ satisfies the following two conditions:

$$
\begin{aligned}
& \forall \varepsilon>0 \exists \delta>0 \text { such that } \\
& \left.\begin{array}{l}
|x|+|y| \leq \delta \\
\forall(x, y) \in \mathbb{R}^{2 k}
\end{array}\right\} \Longrightarrow\left\{\begin{array}{l}
\varphi(t, x, y) \subset \overline{B\left(-m_{1} p(x), \varepsilon(|x|+|y|)\right)} \\
\forall t \in[a, b]
\end{array}\right\} \\
& \forall \varepsilon>0 \exists R>0 \text { such that } \\
& \left.\begin{array}{l}
|x|+|y| \geq R \\
\forall(x, y) \in \mathbb{R}^{2 k}
\end{array}\right\} \Longrightarrow\left\{\begin{array}{l}
\varphi(t, x, y) \subset \overline{B\left(-m_{2} p(x), \varepsilon(|x|+|y|)\right)} \\
\forall t \in[a, b] .
\end{array}\right\}
\end{aligned}
$$

where $m_{1}, m_{2}>0$ are constants.

Theorem 2. Let the map $l: C^{1}\left([a, b], \mathbb{R}^{k}\right) \rightarrow \mathbb{R}^{k} \times \mathbb{R}^{k}$ be given by $(2.2)$, let $\Lambda=\left\{\frac{\mu_{i}}{\eta_{i}}: \eta_{i}>0\right\}$ and let $\varphi:[a, b] \times \mathbb{R}^{k} \times \mathbb{R}^{k} \rightarrow c f\left(\mathbb{R}^{k}\right)$ be a Carathéodory map satisfying $(2.9)-(2.10)$ with constants $m_{1}, m_{2}>0$ such that

$$
\min \left\{m_{1}, m_{2}\right\}<\min \Lambda \leq \max \Lambda<\max \left\{m_{1}, m_{2}\right\} .
$$

Then there exists a non-trivial solution of the Sturm-Liouville problem (2.1).

Proof. Let us denote $m=\min \left\{m_{1}, m_{2}\right\}$ and $M=\max \left\{m_{1}, m_{2}\right\}$, let $\nu>$ $\frac{\max \Lambda}{m}$ be a fixed constant, let $q_{1}, q_{2}:(0,+\infty) \rightarrow[0,+\infty)$ be continuous maps forming a partition of unity associated with the open cover $\{(0,2 \nu),(\nu,+\infty)\}$ of the interval $(0,+\infty)$, and let us define the Carathéodory map

$$
\begin{aligned}
& \psi:[a, b] \times \mathbb{R}^{k} \times \mathbb{R}^{k} \times(0,+\infty) \rightarrow c f\left(\mathbb{R}^{k}\right) \\
& \psi(t, x, y, \lambda)=q_{1}(\lambda) \lambda \varphi(t, x, y)-q_{2}(\lambda) \lambda m_{2} p(x) .
\end{aligned}
$$

Let us now consider the differential inclusion

$$
\left.\begin{array}{rl}
u^{\prime \prime}(t) & \in \psi\left(t, u(t), u^{\prime}(t), \lambda\right) \text { a.e. on }(a, b) \\
l(u) & =0
\end{array}\right\} .
$$

We can see that $(\lambda, u) \in(0,+\infty) \times C^{1}\left([a, b], \mathbb{R}^{k}\right)$ is a solution of this problem if and only if $u \in T \Psi(\lambda, u)$, where

$$
\begin{aligned}
& \Psi:(0,+\infty) \times C^{1}\left([a, b], \mathbb{R}^{k}\right) \rightarrow \operatorname{cf}\left(L^{1}\left((a, b), \mathbb{R}^{k}\right)\right) \\
& \Psi(\lambda, u)=\left\{w \in L^{1}\left((a, b), \mathbb{R}^{k}\right): w(t) \in \psi\left(t, u(t), u^{\prime}(t), \lambda\right) \text { for a.e. } t \in[a, b] .\right\}
\end{aligned}
$$

Let us also observe that, because $\nu>1$, a pair $(1, u)$ is a solution of problem (2.11) if and only if $u$ is a solution of problem (2.1). Consider the map

$$
\begin{aligned}
& f:(0,+\infty) \times C^{1}\left([a, b], \mathbb{R}^{k}\right) \rightarrow c f\left(C^{1}\left([a, b], \mathbb{R}^{k}\right)\right) \\
& f(\lambda, u)=u-T \Psi(\lambda, u)
\end{aligned}
$$


and let

$$
\begin{aligned}
& P: C^{1}\left([a, b], \mathbb{R}^{k}\right) \rightarrow L^{1}\left((a, b), \mathbb{R}^{k}\right) \\
& P(u)(t)=p(u(t))
\end{aligned}
$$

denote the Niemytzki map for the map $p$. The proof of Theorem 2 will be given now in three steps.

Step 1. We are going to show that $\mathcal{B}_{f} \subset\left\{\left(\frac{\lambda}{m_{1}}, 0\right): \lambda \in \Lambda\right\}$. For this let us take a sequence $\left\{\left(\lambda_{n}, u_{n}\right)\right\} \subset(0,+\infty) \times C^{1}\left([a, b], \mathbb{R}^{k}\right)$ of non-trivial solutions of problem (2.11) such that $\lambda_{n} \rightarrow \lambda_{0} \in[0,+\infty)$ and $u_{n} \rightarrow 0$. We have

$$
u_{n} \in q_{1}\left(\lambda_{n}\right) \lambda_{n} T\left(\Phi\left(u_{n}\right)+m_{1} P\left(u_{n}\right)\right)-\lambda_{n} T\left(m_{1} q_{1}\left(\lambda_{n}\right)+m_{2} q_{2}\left(\lambda_{n}\right)\right) P\left(u_{n}\right) .
$$

Let us denote $v_{n}=\frac{u_{n}}{\left\|u_{n}\right\|_{k}}$. Then

$$
v_{n} \in q_{1}\left(\lambda_{n}\right) \lambda_{n} T \frac{\Phi\left(u_{n}\right)+m_{1} P\left(u_{n}\right)}{\left\|u_{n}\right\|_{k}}-\lambda_{n} T\left(\left(m_{1} q_{1}\left(\lambda_{n}\right)+m_{2} q_{2}\left(\lambda_{n}\right)\right) P\left(v_{n}\right)\right) .
$$

By (2.9) we have $\frac{\Phi\left(u_{n}\right)+m_{1} P\left(u_{n}\right)}{\left\|u_{n}\right\|_{k}} \rightarrow\{0\}$ (in the Hausdorff metric). Because the sequence $\left\{\left(m_{1} q_{1}\left(\lambda_{n}\right)+m_{2} q_{2}\left(\lambda_{n}\right)\right) P\left(v_{n}\right)\right\}$ is bounded, there exists a subsequence of $\left\{v_{n}\right\}$ convergent to $v_{0} \in C^{1}\left([a, b], \mathbb{R}^{k}\right)$, where $\left\|v_{0}\right\|_{k}=1$. So letting $n \rightarrow+\infty$ we get $v_{0}=-\lambda_{0} T\left(\left(m_{1} q_{1}\left(\lambda_{0}\right)+m_{2} q_{2}\left(\lambda_{0}\right)\right) P\left(v_{0}\right)\right)$ and

$$
\left.\begin{array}{rl}
v_{0}^{\prime \prime}(t)+\lambda_{0}\left(m_{1} q_{1}\left(\lambda_{0}\right)+m_{2} q_{2}\left(\lambda_{0}\right)\right) p\left(v_{0}(t)\right) & =0 \text { for a.e. } t \in(a, b) \\
l(u) & =0
\end{array}\right\} .
$$

So, by Lemma $3,\left(m_{1} q_{1}\left(\lambda_{0}\right)+m_{2} q_{2}\left(\lambda_{0}\right)\right) \lambda_{0} \in \Lambda$. No matter what is the value of $\lambda_{0}$ we have $m_{1} q_{1}\left(\lambda_{0}\right)+m_{2} q_{2}\left(\lambda_{0}\right) \in[m, M]$. So $\lambda_{0} \leq \frac{\max \Lambda}{m}<\nu$ what implies $m_{1} \lambda_{0} \in \Lambda$ and finishes the proof of Step 1 .

Step 2. We will now show that $s\left[f, \frac{\min \Lambda}{m_{1}}, \frac{\max \Lambda}{m_{1}}\right]=-1$. For this, first let us observe that for $\lambda \notin\left\{\frac{\lambda}{m_{1}}: \lambda \in \Lambda\right\}$ there exists $r>0$ such that by (2.9) the map

$$
f(\lambda, \cdot): \overline{B(0, r)} \rightarrow c f\left(C^{1}\left([a, b], \mathbb{R}^{k}\right)\right)
$$

is homotopic to the map

$$
\begin{aligned}
& \bar{f}(\lambda, \cdot): \overline{B(0, r)} \rightarrow c f\left(C^{1}\left([a, b], \mathbb{R}^{k}\right)\right) \\
& \bar{f}(\lambda, u)=u+\lambda\left(m_{1} q_{1}(\lambda)+m_{2} q_{2}(\lambda)\right) T P(u) .
\end{aligned}
$$

We can see also that the map

$$
\bar{f}(\lambda, \cdot): \overline{B(0, r)} \rightarrow C^{1}\left([a, b], \mathbb{R}^{k}\right)
$$


for $\lambda \geq \nu$ may be joined by homotopy with the map

$$
\begin{aligned}
& f_{0}(\lambda, \cdot): \overline{B(0, r)} \rightarrow C^{1}\left([a, b], \mathbb{R}^{k}\right) \\
& f_{0}(\lambda, u)=u+\lambda m_{1} T P(u) .
\end{aligned}
$$

Let the homotopy

$$
\begin{aligned}
& h:[0,1] \times \overline{B(0, r)} \rightarrow C^{1}\left([a, b], \mathbb{R}^{k}\right) \\
& h(\tau, u)=u+\lambda\left(\tau m_{1} q_{1}(\lambda)+m_{2} \tau q_{2}(\lambda)+(1-\tau) m_{1}\right) T P(u)
\end{aligned}
$$

be given. Similarly to what we showed in Step 1 of this proof, for any nontrivial zero of the homotopy $h$, there must be

$$
\lambda\left(\tau m_{1} q_{1}(\lambda)+m_{2} \tau q_{2}(\lambda)+(1-\tau) m_{1}\right) \in \Lambda
$$

what, having $\left(\tau m_{1} q_{1}(\lambda)+m_{2} \tau q_{2}(\lambda)+(1-\tau) m_{1}\right) \geq(1-\tau) m_{1}+\tau m \geq m$, implies $\lambda \leq \frac{\max \Lambda}{m}$ and contradicts $\lambda \geq \nu$. On the other hand, for $\lambda<\nu$ we have $\bar{f}(\lambda, \cdot)=f_{0}(\lambda, \cdot)$.

Let $r>0$ and $\lambda_{0} \in\left(0, \frac{\min \Lambda}{m_{1}}\right)$ be fixed. We will show that

$$
f_{0}\left(\lambda_{0}, \cdot\right): \overline{B(0, r)} \rightarrow C^{1}\left([a, b], \mathbb{R}^{k}\right)
$$

may be joined by homotopy with the identity map. Let a homotopy be given by $h(\tau, u)=u+\lambda_{0} \tau T m_{1} P(u)$. We can conclude from Lemma 3 that $\left(\lambda_{0} \tau, 0\right) \notin \mathcal{B}_{f}$ for $\tau \in[0,1]$. That is why we have no non-trivial zeros of $h(\tau, u)=0$. Hence, by homotopy property of topological degree, we have $\operatorname{deg}\left(f_{0}\left(\lambda_{0}, \cdot\right), B(0, r), 0\right)=1$.

Assume now that $\lambda_{0} \in\left(\frac{\max \Lambda}{m_{1}},+\infty\right)$ and let $i \in\{1, \ldots, k\}$ be such that $\eta_{i}>0$ and $u_{\mu_{i}}=-\mu_{i} T u_{\mu_{i}}$ with $u_{\mu_{i}, i}(t)>0$ for $t \in(a, b)$ where $u_{\mu_{i}, i}$ is the $i$-th coordinate of $u_{\mu_{i}}$. We will show that for $\lambda_{0}$ the map $f_{0}\left(\lambda_{0}, \cdot\right)$ may be joined by homotopy on $\overline{B(0, r)}$ with the map

$$
\begin{aligned}
& f_{1}: \overline{B(0, r)} \rightarrow C^{1}\left([a, b], \mathbb{R}^{k}\right) \\
& f_{1}(u)=f_{0}\left(\lambda_{0}, u\right)-u_{\mu_{i}} .
\end{aligned}
$$

A homotopy $h:[0,1] \times \overline{B(0, r)} \rightarrow C^{1}\left([a, b], \mathbb{R}^{k}\right)$ is given by

$$
h(\tau, u)=f_{0}\left(\lambda_{0}, u\right)-\tau u_{\mu_{i}} .
$$

Assume now that for $\|u\|_{k} \leq r$ and $\tau \in(0,1]$ the equality $h(\tau, u)=0$ holds and

$$
\begin{aligned}
u+\lambda_{0} m_{1} T P(u)-\tau u_{\mu_{i}} & =0 \\
u+T\left(\lambda_{0} m_{1} P(u)+\tau \mu_{i} u_{\mu_{i}}\right) & =0 .
\end{aligned}
$$


So we have

$$
\left.\begin{array}{rl}
u^{\prime \prime}(t)+\lambda_{0} m_{1} p(u(t))+\tau \mu_{i} u_{\mu_{i}}(t) & =0 \text { for a.e. } t \in(a, b) \\
l(u) & =0
\end{array}\right\}
$$

what, by the maximum principle, gives $u \geq 0$ and, consequently, $p_{i}\left(u_{i}\right)=\eta_{i} u_{i}$. Since $u_{i}=-\lambda_{0} T_{i} m_{1} \eta_{i} u_{i}+\tau u_{\mu_{i}, i}$ and also

$$
\begin{aligned}
\left\langle u_{i}, u_{\mu_{i}, i}\right\rangle & =-\lambda_{0}\left\langle T_{i} m_{1} \eta_{i} u_{i}, u_{\mu_{i}, i}\right\rangle+\tau\left\langle u_{\mu_{i}, i}, u_{\mu_{i}, i}\right\rangle \\
& =-\lambda_{0}\left\langle m_{1} \eta_{i} u_{i}, T_{i} u_{\mu_{i}, i}\right\rangle+\tau\left\langle u_{\mu_{i}, i}, u_{\mu_{i}, i}\right\rangle \\
& =\frac{\lambda_{0} m_{1} \eta_{i}}{\mu_{i}}\left\langle u_{i}, u_{\mu_{i}, i}\right\rangle+\tau\left\langle u_{\mu_{i}, i}, u_{\mu_{i}, i}\right\rangle
\end{aligned}
$$

we have

$$
\frac{\mu_{i}-m_{i} \eta_{i} \lambda_{0}}{\mu_{i}}\left\langle u_{i}, u_{\mu_{i}, i}\right\rangle=\tau\left\langle u_{\mu_{i}, i}, u_{\mu_{i}, i}\right\rangle>0 .
$$

Because $u_{\mu_{i}, i} \geq 0$ and $u_{i} \geq 0$, it must be also $\mu_{i}>m_{1} \eta_{i} \lambda_{0}$ what contradicts the assumption $\lambda_{0}>\frac{\max \Lambda}{m_{1}} \geq \frac{\mu_{i}}{\eta_{i} m_{1}}$.

If $\tau=0$, then $h(\tau, \cdot)=f_{0}\left(\lambda_{0}, \cdot\right)$ and $h(0, u)=0$ if and only if $f_{0}\left(\lambda_{0}, u\right)=0$. Because $m \lambda_{0} \notin \Lambda, f_{0}\left(\lambda_{0}, u\right)=0$ implies $u=0$. Hence the homotopy $h$ has no non-trivial zeroes. Also, $h(1, \cdot)$ has no zeroes at all and that is why $\operatorname{deg}\left(f_{0}\left(\lambda_{0}, \cdot\right), B(0, r), 0\right)=0$. So Step 2 is proved.

Step 3. Let us observe that by Theorem 1 there exists a non-compact component $\mathcal{C} \subset \mathcal{R}_{f}$. Now we are going to show that there exists a sequence $\left\{\left(\lambda_{n}, u_{n}\right)\right\} \subset \mathcal{C}$ such that $\left\|u_{n}\right\|_{k} \rightarrow+\infty$ and $\lambda_{n} \rightarrow \lambda_{0} \in\left\{\frac{\lambda}{m_{2}}: \lambda \in \Lambda\right\}$.

Because the set $\mathcal{C}$ is not compact, there exists a sequence $\left\{\left(\lambda_{n}, u_{n}\right)\right\} \subset \mathcal{C}$ such that $\lambda_{n} \rightarrow 0$, or $\lambda_{n} \rightarrow+\infty$, or $\left\|u_{n}\right\|_{k} \rightarrow+\infty$. We are going to show that there must be $\left\|u_{n}\right\|_{k} \rightarrow+\infty$.

First, let us assume that $\lambda_{n} \rightarrow 0$ and that $\left\{\left\|u_{n}\right\|_{k}\right\}$ is bounded. In this case, for almost all $n \in \mathbb{N}$, the relation $u_{n} \in \lambda_{n} T \Phi\left(u_{n}\right)$ holds and consequently $u_{n} \rightarrow 0$. As we showed in Step 1, $u_{n} \rightarrow 0$ and $\lambda_{n} \rightarrow \lambda_{0}$ implies that $\lambda_{0} \in$ $\left\{\frac{\lambda}{m_{1}}: \lambda \in \Lambda\right\}$ what contradicts $\lambda_{n} \rightarrow 0$.

Now let us consider the case $\lambda_{n} \rightarrow+\infty$. Then, for almost all $n \in \mathbb{N}$, if $u_{n} \neq 0$, then there must be $q_{2}\left(\lambda_{n}\right)=1$ and $u_{n}=\lambda_{n} T m_{2} P\left(u_{n}\right)$. By Lemma 3 there is $\lambda_{n} \in\left\{\frac{\lambda}{m_{2}}: \lambda \in \Lambda\right\}$ what contradicts $\lambda_{n} \rightarrow+\infty$.

So we may assume that $\left\|u_{n}\right\|_{k} \rightarrow+\infty$ and $\lambda_{n} \rightarrow \lambda_{0} \in(0,+\infty)$. Now we are going to prove that in such situation $\lambda_{0} \in\left\{\frac{\lambda}{m_{2}}: \lambda \in \Lambda\right\}$. Indeed, we can see that

$$
u_{n} \in\left\{\begin{array}{l}
\lambda_{n} q_{1}\left(\lambda_{n}\right) T\left(\Phi\left(u_{n}\right)+m_{2} P\left(u_{n}\right)\right)-\lambda_{n} T m_{2} P\left(u_{n}\right) \\
\lambda_{n} q_{1}\left(\lambda_{n}\right) T \frac{\Phi\left(u_{n}\right)+m_{2} P\left(u_{n}\right)}{\left\|u_{n}\right\|_{k}}-\lambda_{n} T m_{2} P\left(v_{n}\right)
\end{array}\right.
$$


where $v_{n}=\frac{u_{n}}{\left\|u_{n}\right\|_{k}}$. We are going to show that for all $\varepsilon>0$ there exists $N \in \mathbb{N}$ such that

$$
T \frac{\Phi\left(u_{n}\right)+m_{2} P\left(u_{n}\right)}{\left\|u_{n}\right\|_{k}} \subset \overline{B(0, \varepsilon)} \quad(n>N) .
$$

For this, let $\varepsilon>0$ be fixed. By (2.10) there exists $R>0$ such that for $\left|u_{n}(t)\right|+\left|u_{n}^{\prime}(t)\right| \geq R$ the relation

$$
\frac{\varphi\left(t, u(t), u^{\prime}(t)\right)+m_{2} p(u(t))}{|u(t)|+\left|u^{\prime}(t)\right|} \subset B(0, \varepsilon)
$$

holds. Let $m_{R} \in L^{1}(a, b)$ be an integrable function such that

$$
\left\{\begin{array}{l}
\forall w \in L^{1}\left((a, b), \mathbb{R}^{k}\right) \\
\forall x \in \mathbb{R}^{k} \\
\forall y \in \mathbb{R}^{k} \\
\forall t \in[a, b]
\end{array}\right\}:\left\{\begin{array}{l}
|x|+|y| \leq R \\
w(t) \in \varphi(t, x, y)
\end{array}\right\} \Rightarrow|w(t)| \leq m_{R}(t) .
$$

Let us now take any $w \in L^{1}\left((a, b), \mathbb{R}^{k}\right)$ such that

$$
w(t) \in \frac{\varphi\left(t, u_{n}(t), u_{n}^{\prime}(t)\right)+m_{2} p\left(u_{n}(t)\right)}{\left\|u_{n}\right\|_{k}} \quad(t \in[a, b])
$$

and consider the two situations

$$
\begin{aligned}
& \left|u_{n}(t)\right|+\left|u_{n}^{\prime}(t)\right| \leq R \\
& \left|u_{n}(t)\right|+\left|u_{n}^{\prime}(t)\right|>R .
\end{aligned}
$$

For them we have respectively $|w(t)| \leq \frac{m_{R}(t)}{\left\|u_{n}\right\|_{k}}$ and

$$
\begin{aligned}
\frac{\varphi(t,}{\left.u_{n}(t), u_{n}^{\prime}(t)\right)+m_{2} p\left(u_{n}(t)\right)} & \\
& =\frac{\varphi\left(t, u_{n}(t), u_{n}^{\prime}(t)\right)+m_{2} p\left(u_{n}(t)\right)}{\left|u_{n}(t)\right|+\left|u_{n}^{\prime}(t)\right|} \cdot \frac{\left|u_{n}(t)\right|+\left|u_{n}^{\prime}(t)\right|}{\left\|u_{n}\right\|_{k}} \\
|w(t)| & \in \frac{\varphi\left(t, u_{n}(t), u_{n}^{\prime}(t)\right)+m_{2} p\left(u_{n}(t)\right)}{\left|u_{n}(t)\right|+\left|u_{n}^{\prime}(t)\right|} \cdot \frac{\left|u_{n}(t)\right|+\left|u_{n}^{\prime}(t)\right|}{\left\|u_{n}\right\|_{k}} \\
& \subset B(0, \varepsilon) .
\end{aligned}
$$

So for $n \in \mathbb{N}$ big enough and any $t \in[a, b]$ we have $|w(t)|<\max \left\{\varepsilon, \frac{m_{R}(t)}{\left\|u_{n}\right\|_{k}}\right\}$ what shows that

$$
T \frac{\Phi\left(u_{n}\right)+m_{2} P\left(u_{n}\right)}{\left\|u_{n}\right\|_{k}} \subset B(0, \varepsilon\|T\|(b-a))
$$


with $\|T\|$ denoting the norm of the map $T: L^{1}\left((a, b), \mathbb{R}^{k}\right) \rightarrow C^{1}\left([a, b], \mathbb{R}^{k}\right)$.

Let us observe that, because of the compactness of $T$, we may assume that $v_{n} \rightarrow v_{0}$, where $v_{0} \neq 0$. Hence, letting $n \rightarrow+\infty$ we get $v_{0}=-\lambda_{0} T m_{2} P\left(v_{0}\right)$ what results in $\lambda_{0} \in\left\{\frac{\lambda}{m_{2}}: \lambda \in \Lambda\right\}$. Further, let us observe that the assumptions of the theorem imply that $\left\{\frac{\lambda}{m}: \lambda \in \Lambda\right\} \subset(1,+\infty)$ and $\left\{\frac{\lambda}{M}: \lambda \in \Lambda\right\} \subset$ $(0,1)$. As a consequence of Steps 1 and 3 of this proof we can see that the connected set $\mathcal{C}$ contains pairs $\left(\lambda_{1}, u\right)$ and $\left(\lambda_{2}, u\right)$ with $\lambda_{1}<1$ and $\lambda_{2}>1$. That is why we can conclude that there exists $(1, u) \in \mathcal{C}$. For such a solution of the inclusion $0 \in f(\lambda, u)$ there must be $u \neq 0$ because $(1,0) \notin \mathcal{R}_{f}$.

\section{Examples and remarks}

In this section we will give some applications of Theorem 2 to the convexvalued boundary value problems

$$
\left.\begin{array}{rl}
u^{\prime \prime}(t) & \in \varphi\left(t, u(t), u^{\prime}(t)\right) \text { for a.e. } t \in(0,1) \\
u(0) & =u(1)=0 \\
u^{\prime \prime}(t) & \in \varphi\left(t, u(t), u^{\prime}(t)\right) \text { for a.e. } t \in(0,1) \\
u(0) & =u^{\prime}(1)=0
\end{array}\right\}
$$

Let us remind that the topological transversality method of Granas and a priori bounds technique have been used to existence theorems for the above second order differential equations (inclusions) $[6,7,10,11]$. The fundamental assumption there, which guaranteed the bound of zeros of the homotopy joining suitable vector fields associated with the boundary value problem, were the following Bernstein conditions:

(H1) There exists a constant $R>0$ such that if $\left|x_{0}\right|>R$ and $y_{0} \in \mathbb{R}^{k}$, then there is a $\delta>0$ such that

$$
\underset{t \in[a, b]}{\operatorname{essinf}} \inf \left\{\langle x, w\rangle+|y|^{2}: w \in \varphi(t, x, y),(x, y) \in B\left(\left(x_{0}, y_{0}\right), \delta\right)\right\}>0
$$

where $B\left(\left(x_{0}, y_{0}\right), \delta\right)=\left\{(x, y) \in \mathbb{R}^{k} \times \mathbb{R}^{k}:\left|x-x_{0}\right|+\left|y-y_{0}\right|<\delta\right\}$.

(H2) There is a function $\Phi:[0,+\infty) \rightarrow[0,+\infty)$ such that the function $s \rightarrow \frac{s}{\Phi(s)}$ is in $L_{l o c}^{\infty}[0,+\infty), \int_{0}^{+\infty} \frac{s}{\Phi(s)} d s=+\infty,|\varphi(t, x, y)| \leq \Phi(y)$ for a.e. $t \in[a, b]$ and all $(x, y)$ with $|x|+|y| \leq R$ where $R$ is given in condition (H1).

(H3) There exist constants $k, \alpha>0$ such that $|\varphi(t, x, y)| \leq 2 \alpha(\langle x, w\rangle+$ $\left.|y|^{2}\right)+k$ for a.e. $t \in[a, b]$, all $(x, y)$ with $|x|+|y| \leq R$ and $w \in \varphi(t, x, y)$. 
Below we will give some ordinary differential inclusions, for which the orientors $\varphi(t, x, y)$ locally have linear asymptotics "at zero and at infinity" (also all assumptions of Theorem 2 are satisfied), but they do not satisfy the above Bernstein conditions (H1) - (H3).

Corollary 1. Let $\varphi:[0,1] \times \mathbb{R}^{k} \times \mathbb{R}^{k} \rightarrow c f\left((-\infty, 0]^{k}\right)$ be a Carathéodory map satisfying $(2.9)-(2.10)$ with constants $m_{1}, m_{2}>0$ such that

$$
\min \left\{m_{1}, m_{2}\right\}<\min \left\{\frac{\pi^{2}}{\eta_{i}}: \eta_{i}>0\right\} \leq \max \left\{\frac{\pi^{2}}{\eta_{i}}: \eta_{i}>0\right\}<\max \left\{m_{1}, m_{2}\right\} .
$$

Then there exists a non-trivial solution of problem (3.1).

Proof. Let us observe that the only eigenvalue of the problem

$$
\left.\begin{array}{r}
u^{\prime \prime}(t)+\lambda u(t)=0 \\
u(0)=u(1)=0
\end{array}\right\}
$$

for which there exists a non-negative eigenvector, is $\mu_{0}=\pi^{2}$. Then $\varphi$ satisfies all assumptions of Theorem 2. So there exists a non-trivial solution of problem (3.1).

Remark 2. The multi-valued map $\varphi$ given in Corollary 1 does not satisfy condition (H1). Indeed, let us take large $x_{0} \in[0,+\infty)^{k}$ and $y_{0}=0$. Then, if $w \in \varphi(t, x, y)$ then $w<0$. So $\langle x, w\rangle+|y|^{2}<0$ and condition (H1) is not satisfied.

Corollary 2. Let $\varphi:[0,1] \times \mathbb{R}^{k} \times \mathbb{R}^{k} \rightarrow c f\left(\mathbb{R}^{k}\right)$ be a Carathéodory map satisfying $(2.9)-(2.10)$ with constants $m_{1}, m_{2}>0$ such that

$$
\min \left\{m_{1}, m_{2}\right\}<\min \left\{\frac{\pi^{2}}{\eta_{i}}: \eta_{i}>0\right\} \leq \max \left\{\frac{\pi^{2}}{\eta_{i}}: \eta_{i}>0\right\}<\max \left\{m_{1}, m_{2}\right\} .
$$

Assume additionally that, for each $M>0, \mu(\{t:|\varphi(t, 0, y)|>M\})>0 \quad(\mu$ denotes the Lebesgue measure) where $k<|y|<K$ for $k, K>0$. Then there exists a non-trivial solution of problem (3.1).

Proof. Let us observe that the map $\varphi$ satisfies all assumptions of Theorem 2. So there exists a non-trivial solution of problem (3.1).

Remark 3. The multi-valued map $\varphi$ given in Corollary 2 does not satisfy condition (H2). Indeed, let us observe that there is no function $\Phi$ such that $|\varphi(t, x, y)| \leq \Phi(y)$ for a.e. $t \in[0,1]$ and all $(x, y)$ such that $|x|+|y| \leq R$. So condition (H2) is not satisfied. 
Corollary 3. Let $\varphi:[0,1] \times \mathbb{R}^{k} \times \mathbb{R}^{k} \rightarrow c f\left((-\infty, 0]^{k}\right)$ be a Carathéodory map satisfying $(2.9)-(2.10)$ with constants $m_{1}, m_{2}>0$ such that

$$
\min \left\{m_{1}, m_{2}\right\}<\min \left\{\frac{\pi^{2}}{4 \eta_{i}}: \eta_{i}>0\right\} \leq \max \left\{\frac{\pi^{2}}{4 \eta_{i}}: \eta_{i}>0\right\}<\max \left\{m_{1}, m_{2}\right\} .
$$

Then there exists a non-trivial solution of problem (3.2).

Proof. Let us observe that the only eigenvalue of the problem

$$
\left.\begin{array}{r}
u^{\prime \prime}(t)+\lambda u(t)=0 \\
u(0)=u^{\prime}(1)=0
\end{array}\right\}
$$

for which there exists a non-negative eigenvector, is $\mu_{0}=\frac{\pi^{2}}{4}$. Then the map $\varphi$ satisfies all assumptions of Theorem 2. So there exists a non-trivial solution of problem (3.1).

Remark 4. The multi-valued map $\varphi$ given in Corollary 3 does not satisfy condition (H1). Indeed, let us take large $x_{0} \in[0,+\infty)^{k}$ and $y_{0}=0$. Then, if $w \in \varphi(t, x, y)$ then $w<0$. So $\langle x, w\rangle+|y|^{2}<0$ and condition (H1) is not satisfied.

Corollary 4. Let $\varphi:[0,1] \times \mathbb{R}^{k} \times \mathbb{R}^{k} \rightarrow c f\left(\mathbb{R}^{k}\right)$ be a Carathéodory map satisfying $(2.9)-(2.10)$ with constants $m_{1}, m_{2}>0$ such that

$$
\min \left\{m_{1}, m_{2}\right\}<\min \left\{\frac{\pi^{2}}{4 \eta_{i}}: \eta_{i}>0\right\} \leq \max \left\{\frac{\pi^{2}}{4 \eta_{i}}: \eta_{i}>0\right\}<\max \left\{m_{1}, m_{2}\right\}
$$

Additionally, assume that, for each $M>0, \mu(\{t:|\varphi(t, 0, y)|>M\})>0$ (Lebesgue measure), where $k<|y|<K$ for $k, K>0$. Then there exists a non-trivial solution of problem (3.2).

Proof. Let us observe that the map $\varphi$ satisfies all assumptions of Theorem 2 . So there exists a non-trivial solution of problem (3.2).

Remark 5. The multi-valued map $\varphi$ given in Corollary 4 does not satisfy condition (H2). Indeed, let us observe that there is no function $\Phi$ such that $|\varphi(t, x, y)| \leq \Phi(y)$ for a.e. $t \in[0,1]$ and all $(x, y)$ such that $|x|+|y| \leq R$. So condition (H2) is not satisfied.

Remark 6. In [5] a special case of problem (2.1) was considered where $\alpha_{i}$ and $\beta_{i}$ are constant (do not depend on $i \in\{1, \ldots, k\}$ ) and $\varphi:[a, b] \times \mathbb{R}^{k} \times \mathbb{R}^{k} \rightarrow$ $\operatorname{cl}\left(\mathbb{R}^{k}\right)$ is a Carathéodory map satisfying the linear growth condition

$$
|\varphi(t, x, y)| \leq w_{0}(t)+w_{1}(t)|x|+w_{2}(t)|y|
$$


for integrable functions $w_{0}, w_{1}, w_{2} \in L^{1}(a, b)$. Let us now denote by $G$ : $[a, b]^{2} \rightarrow \mathbb{R}$ the Green function related with the linear problem (2.7). In [5] it is proved that if $w_{1}, w_{2}$ in (3.3) are integrable functions and the map

$$
\begin{gathered}
L: C\left([a, b], \mathbb{R}^{k}\right) \times C\left([a, b], \mathbb{R}^{k}\right) \rightarrow C\left([a, b], \mathbb{R}^{k}\right) \times C\left([a, b], \mathbb{R}^{k}\right) \\
L(\xi, \eta)=\left(\int_{a}^{b}|G(\cdot, s)|\left[w_{1}(s) \xi(s)+w_{2}(s) \eta(s)\right] d s,\right. \\
\left.\quad \int_{a}^{b}\left|G_{t}(\cdot, s)\right|\left[w_{1}(s) \xi(s)+w_{2}(s) \eta(s)\right] d s\right)
\end{gathered}
$$

has spectral radius $r(L)<1$, then problem (2.1) has a solution.

In the special case of $w_{2}=0, w_{1}$ constant and Dirichlet boundary conditions $l(u)=(u(a), u(b))$, condition $r(L)<1$ is equivalent to $w_{1}<\frac{\pi^{2}}{(b-a)^{2}}$ (see [5: Example 12.2]). Let us now again consider $\varphi:[0,1] \times \mathbb{R}^{2} \times \mathbb{R}^{2} \rightarrow \mathbb{R}^{2}$ given in Corollary 1 , with $\eta_{i}=1 \quad(i=1, \ldots, k)$, satisfying additionally $|\varphi(t, x, y)| \leq w_{0}+w_{1}|x|$ with $w_{0}, w_{1} \in(0,+\infty)$. In this case, because of (2.9) - (2.10), there must be $w_{1}>\pi^{2}$. So the condition $w_{1}<\frac{\pi^{2}}{(b-a)^{2}}$ is not satisfied and the mentioned theorem given in [5] cannot be applied.

Acknowledgement. The authors are grateful to Prof. Tadeusz Pruszko for inspiration and help during the preparation of this article.

\section{References}

[1] Alexander, J. C. and P. M. Fitzpatrick: Global bifurcation for solutions of equations involving several parameter multivalued condensing mappings. Lect. Notes Math. 886 (1981), 1 - 19.

[2] Borisovic, U. G., Gelman, B. D., Myskis, A. D. and V. V. Obuhowskii: Topological methods in fixed point theory of multivalued mappings (in Russian.) Uspiehi Mat. Nauk 1 (1980), $59-126$.

[3] Cellina, A. and A. Lasota: A new approach to the definition of topological degree for multivalued mappings. Accad. Naz. Lincei 47 (1969), $434-440$.

[4] Chow, S.-N. and J. K. Hale: Methods of Bifurcation Theory (Grundlehren der math. Wiss.: Vol. 251). New York et al.: Springer-Verlag 1982.

[5] Deimling, K.: Multivalued Differential Equations. Berlin: Walter de Gruyter 1992.

[6] Erbe, L. H. and W. Krawcewicz: Nonlinear boundary value problems for differential inclusions $y^{\prime \prime} \in F\left(t, y, y^{\prime}\right)$. Ann. Polonici Math. 54 (1991), $195-$ 226.

[7] Gaines, R. E. and J. Mawhin: Coincidence Degree and Nonlinear Equations. Lect. Notes Math. 568 (1977). 
[8] Granas, A.: Sur la notion du degré topologique pour une certain classe de transformations dans les espaces de Banach. Bull. Acad. Polon. Sci. 7 (1959), 191 $-194$.

[9] Granas, A. and J. Dugundji: Fixed Point Theory (Springer Monographs in Mathematics). New York: Springer-Verlag 2003.

[10] Granas, A., Guenther, R. B. and J. W. Lee: On a theorem of S. Bernstein. Pacific J. Math. 73 (1977), $67-82$.

[11] Granas, A., Guenther, R. B. and J. W. Lee: Nonlinear Boundary Value Problems for some Classes of Ordinary Differential Equations. Diss. Math. 244 (1985).

[12] Gulgowski, J.: A global bifurcation theorem with applications to nonlinear P $i$ card problems. Nonlin. Anal. 41 (2000), $787-801$.

[13] Hartman, P.: Ordinary Differential Equations. Boston et al.: Birkhäuser Verlag 1982.

[14] Ize, J.: Bifurcation Theory for Fredholm Operators. Memoirs Amer. Math. Soc. 174 (1976).

[15] Ize, J.: Topological Bifurcation. In: Topological Nonlinear Analysis: Degree, Singularity and Variations (eds.: M. Matzeu and A. Vignoli; Progress in Nonlin. Diff. Equ. \& Their Appl.: Vol. 15). Boston: Birkhäuser Verlag 1995, pp. 341 $-463$.

[16] Krasnoselskii, M. A.: Topological Methods in the Theory of Nonlinear Integral Equations. Chapter 2. New York: Macmillan 1965.

[17] Lasry, J. M. and R. Robert: Analyse non linéaire multivoque. Report No.7611. Paris: Centre de Recherche de Math. de la Décision, Université de ParisDauphine.

[18] Le, V. K. and K. Schmitt: Global Bifurcation in Variational Inequalities. New York: Springer-Verlag 1997.

[19] Ma, T. W.: Topological Degrees for Set-Valued Compact Fields in Locally Convex Spaces. Diss. Math. 92. Warszawa: PWN 1972.

[20] Nirenberg, L.: Topics in Nonlinear Functional Analysis. New York: Courant Inst. of Math. Sci., New York Univ. 1974.

[21] Protter, M. H. and H. F. Weinberger: Maximum Principles in Differential Equations. New York: Springer-Verlag 1984.

[22] Pruszko, T.: Some applications of the topological degree theory to multi-valued boundary value problems. Diss. Math. 229. Warszawa: PWN 1984, $1-52$.

[23] Rabinowitz, P.: Some global results for nonlinear eigenvalue problems. J. Funct. Anal. 7 (1971), 487 - 513.

[24] Whyburn, G. and E. Duda: Dynamic Topology. New York: Springer-Verlag 1979.

Received 24.07.2003; in revised form 24.11.2003 